\title{
On the strong convergence to equilibrium of the Foias solutions of the transport equation
}

\author{
by JAN MALCZAK (Kraków)
}

\begin{abstract}
We define the Foias solutions of the transport equation and we prove that the strong asymptotic stability of the Foiaş solutions is equivalent to the asymptotic stability of the solutions of the transport equation in $L^{1}$.
\end{abstract}

Introduction. The purpose of this paper is to study the behavior of the Foias solutions of the integro-differential equation of the form

$$
\frac{\partial u(t, x)}{\partial t}+\sum_{i=1}^{n} \frac{\partial}{\partial x_{i}}\left(F_{i}(x) u(t, x)\right)+u(t, x)=\int_{X} k(x, y) u(t, y) d y
$$

$t \geq 0, x \in X=\mathbb{R}_{+}^{n}=[0, \infty)^{n}$, where $k: X \times X \rightarrow \mathbb{R}$ is a measurable stochastic kernel, i.e. $k(x, y) \geq 0, \int_{X} k(x, y) d x=1$ for $y \in X$, with the initial value

$$
u(0, x)=f(x) .
$$

This equation generates a semigroup of Markov operators on the space $L^{1}(X)$ given by

$$
T^{t} f(x)=u(t, x)
$$

where $f \in L^{1}(X)$ is the initial value.

The semigroup $\left\{T^{t}\right\}_{t \geq 0}$ describes the evolution in time of the initial density $f \in L^{1}(X)$ appearing in (0.2). The asymptotic behavior of this semigroup in $L^{1}$ was studied in [DłLa] and [Klac].

The following questions arise:

$1^{\circ}$ Can we define a solution of $(0.1)$ if the initial value $(0.2)$ is not an $L^{1}(X)$ function but a finite measure defined on the Borel subsets of $X$ in such a way that this solution will coincide with $\left\{T^{t} f\right\}_{t \geq 0}$ if the initial

1991 Mathematics Subject Classification: 45K05, 45M10.

Key words and phrases: transport equation, Foiaş solution, Markov operator, asymptotic stability. 
measure is absolutely continuous with respect to the Lebesgue measure with Radon-Nikodym derivative $f$ ?

$2^{\circ}$ What kind of dependence does there exist between the statistical behavior of those two kinds of solutions?

We give an answer to the first question by defining the Foias solution for $(0.1)$ in the case where the initial value in $(0.2)$ is a finite measure. Further, we prove that the asymptotic stability of the semigroup $(0.3)$ in $L^{1}(X)$ is equivalent to the strong asymptotic stability of the Foiaş solutions in the sense of the convergence of the total variation of measures. This is an answer to the second question.

Sections 1-3 provide a mathematical base for Section 6 ; however, Theorem 3.1 stated in Section 3 is of independent interest. In Sections 4 and 5 we give a precise description of the semigroups generated by equation $(0.1)$, while Section 6 contains the main results of the paper.

1. A Markov operator on $L^{1}$. Let $(X, \Sigma, \lambda)$ be a $\sigma$-finite measure space. In the sequel we deal exclusively with real-valued functions and measures. Inequalities (equalities) between functions or sets are in the a.e. sense. A linear operator $T: L^{1}(\lambda) \rightarrow L^{1}(\lambda)$ is called a Markov operator if $T(D) \subset D$, where

$$
D=\left\{f \in L^{1}(\lambda): f \geq 0,\|f\|_{1}=1\right\}
$$

is the set of densities and \|\|$_{1}$ stands for the norm in $L^{1}(\lambda)$. For a given Markov operator on $L^{1}(\lambda)$ define a linear operator $U: L^{\infty}(\lambda) \rightarrow L^{\infty}(\lambda)$ to be the adjoint of $T$ :

$$
\langle T u, f\rangle=\langle u, U f\rangle, \quad u \in L^{1}(\lambda), f \in L^{\infty}(\lambda),
$$

Then $U$ satisfies the following conditions:

(i) If $f \in L^{\infty}(\lambda), f \geq 0$ then $U f \geq 0$.

(ii) $U 1=1$.

(iii) If $f_{n} \downarrow 0$ then $U f_{n} \downarrow 0$.

Conditions (i) and (ii) are immediate. For (iii), let $f_{n} \downarrow 0$ and $0 \leq u \in$ $L^{1}(\lambda)$; then

$$
\left\langle u, \lim U f_{n}\right\rangle:=\int u\left(\lim U f_{n}\right) d \lambda=\lim \left\langle u, U f_{n}\right\rangle=\lim \left\langle T u, f_{n}\right\rangle=0 .
$$

Thus $\lim U f_{n}=0$.

2. A Markov operator on measures. Let $X$ be a locally compact metric space. Assume also that every open subset of $X$ is $\sigma$-compact, i.e. the union of a countable family of compact subsets of $X$. Denote by $\mathcal{B}=\mathcal{B}(X)$ the $\sigma$-algebra of Borel subsets of $X$. A measure $\mu: \mathcal{B} \rightarrow \mathbb{R}_{+}$will be called locally finite if it is finite on every compact subset of $X$. Of course in a 
$\sigma$-compact metric space every locally finite measure $\mu$ is $\sigma$-finite, since $X$ may be written as a countable union of compact subsets. The space of all locally finite measures on $X$ will be denoted by $\mathcal{M}=\mathcal{M}(X)$. The subspaces of $\mathcal{M}$ of all finite and all probabilistic measures will be denoted by $\mathcal{M}_{\text {fin }}$ and $\mathcal{M}_{1}$ respectively.

Let a Markov operator $T: L^{1}(X, \mathcal{B}, \lambda) \rightarrow L^{1}(X, \mathcal{B}, \lambda)$ be given. Define $U: L^{\infty}(X, \mathcal{B}, \lambda) \rightarrow L^{\infty}(X, \mathcal{B}, \lambda)$ by (1.1). Then $U$ satisfies conditions (i)-(iii). Finally, assume that $U$ satisfies a Feller type condition

(iv) $U C_{0}(X) \subset C(X)$.

Here $C(X)$ is the space of all continuous bounded functions on $X$ and $C_{0}(X)$ is the space of all continuous functions with compact supports.

Now for $\mu \in \mathcal{M}_{\text {fin }}$ consider the linear functional

$$
h \mapsto \int U h d \mu, \quad h \in C_{0}(X) .
$$

By the Riesz representation theorem there is a unique regular measure, denoted by $P \mu \in \mathcal{M}$, satisfying

$$
\langle h, P \mu\rangle:=\int h d(P \mu)=\int U h d \mu, \quad h \in C_{0}(X) .
$$

In general, except some trivial cases like $A=\emptyset$ or $A=X$, the characteristic function $1_{A}$ is not continuous and $\left\langle 1_{A}, P \mu\right\rangle$ cannot be defined explicitly, but in our case we have the following

Proposition 2.1. Let $(X, \mathcal{B}, \lambda)$ and $T, U$ be as above. Then

$$
P \mu(A)=\left\langle U 1_{A}, \mu\right\rangle \quad \text { for } A \in \mathcal{B} \text {. }
$$

Proof. Let $A \in \Sigma$ be an open set. There exists a sequence $\left\{h_{n}\right\}, h_{n} \in$ $C_{0}(X)$, such that $h_{n} \uparrow 1_{A}$. By $(2.1)$ we have

$$
\left\langle h_{n}, P \mu\right\rangle=\left\langle U h_{n}, \mu\right\rangle \text {. }
$$

By (iii) and the Lebesgue monotone convergence theorem

$$
\left\langle 1_{A}, P \mu\right\rangle=\left\langle U 1_{A}, \mu\right\rangle .
$$

Substituting $A=X$ in (2.2) and using (ii) we have

$$
P \mu(X)=\left\langle 1_{X}, P \mu\right\rangle=\langle U 1, \mu\rangle=\langle 1, \mu\rangle=\mu(X) .
$$

Therefore (2.2) is true for any closed set $F \in \Sigma$, and hence for any $A \in \Sigma$ since $P \mu$ is regular.

Formula (2.2) defines a Markov operator on $\mathcal{M}_{\text {fin }}$. This means that

(I) $P\left(\alpha_{1} \mu_{1}+\alpha_{2} \mu_{2}\right)=\alpha_{1} P \mu_{1}+\alpha_{2} P \mu_{2}$ for $\alpha_{1}, \alpha_{2} \geq 0, \mu_{1}, \mu_{2} \in \mathcal{M}_{\text {fin }}$,

(II) $P \mu(X)=\mu(X)$ for $\mu \in \mathcal{M}_{\text {fin }}$.

Suppose now that $\mu \in \mathcal{M}_{\text {fin }}$ is absolutely continuous with respect to $\lambda$ $(A \in \Sigma, \lambda(A)=0 \Rightarrow \mu(A)=0)$. Note that if $\lambda(A)=0$ then $1_{A}=0$ 
in $L^{\infty}(\lambda)$ sense, thus $U 1_{A}=0 \lambda$-a.e., hence $U 1_{A}=0 \mu$-a.e. The formula (2.2) implies, in turn, that $P \mu(A)=0$. Hence the measure $P \mu$ is absolutely continuous with respect to $\lambda$.

Using the Radon-Nikodym theorem we may define a linear operator $\widehat{P}$ on $L^{1}(X, \mathcal{B}, \lambda)=L^{1}(\lambda)$ as follows. Let $0 \leq u \in L^{1}(\lambda)$, put $d \mu=u d \lambda$; then

$$
\widehat{P} u=\frac{d(P \mu)}{d \lambda} .
$$

Now equation (2.2) means

$$
\left\langle\widehat{P} u, 1_{A}\right\rangle=\left\langle u, U 1_{A}\right\rangle
$$

where $\langle u, f\rangle=\int u \cdot f d \lambda$ for $u \in L^{1}(\lambda), f \in L^{\infty}(\lambda)$. Using linearity and continuity of $U\left(\|U\|_{\infty}=1\right)$ on $L^{\infty}(\lambda)$ we conclude

$$
\langle\widehat{P} u, f\rangle=\langle u, U f\rangle, \quad f \in L^{\infty}(\lambda) .
$$

Now note that $\widehat{P}$ is a Markov operator on $L^{1}(\lambda)$. By (2.5) and (1.1) we have

$$
\widehat{P}=T
$$

3. Strong convergence for measures. Let $(X, \mathcal{B}, \lambda)$ be as in Section 2 . Let $\left\{\mu_{n}\right\}$ be a sequence of finite measures and $\mu \in \mathcal{M}_{\text {fin }}$. We say that $\left\{\mu_{n}\right\}$ converges strongly to $\mu$ if

$$
\lim _{n \rightarrow \infty}\left\|\mu_{n}-\mu\right\|=0
$$

where

$$
\left\|\mu_{n}-\mu\right\|=\sup \sum_{i=1}^{m}\left|\mu_{n}\left(X_{i}\right)-\mu\left(X_{i}\right)\right|
$$

and the supremum is taken over all possible measurable partitions $\left(X_{1}, \ldots\right.$ $\ldots, X_{m}$ ) of $X$ (with arbitrary $m$ ). In the case when the measures $\mu_{n}$ and $\mu$ are absolutely continuous with respect to $\lambda$ with Radon-Nikodym derivatives $f_{n}$ and $f$ respectively we have

$$
\mu_{n}\left(X_{i}\right)-\mu\left(X_{i}\right)=\int_{X_{i}}\left(f_{n}-f\right) d \lambda .
$$

Substituting this into (3.2) we obtain immediately

$$
\left\|\mu_{n}-\mu\right\|=\int_{X}\left|f_{n}-f\right| d \lambda=\left\|f_{n}-f\right\|_{1} .
$$

The value $\|\mu\|=\mu(X)$ is called the norm of $\mu \in \mathcal{M}_{\text {fin }}$.

Let $T: L^{1}(\lambda) \rightarrow L^{1}(\lambda)$ be a Markov operator. We say that $\left\{T^{n}\right\}$ is asymptotically stable if there exists a unique $f_{*} \in D$ such that $T f_{*}=f_{*}$ and

$$
\lim \left\|T^{n} f-f_{*}\right\|_{1}=0 \quad \text { for every } f \in D .
$$


The operator $P$ defined by (2.1) is strongly asymptotically stable if there is a unique measure $\mu_{*} \in \mathcal{M}_{1}$ such that $P \mu_{*}=\mu_{*}$ and $\left\{P^{n} \mu\right\}$ converges strongly to $\mu_{*}$ for every $\mu \in \mathcal{M}_{1}$.

Finally, for every $\mu \in \mathcal{M}$ we have the Lebesgue decomposition $\mu=$ $\mu_{\mathrm{a}}+\mu_{\mathrm{s}}$, where $\mu_{\mathrm{a}}$ is the maximal measure absolutely continuous with respect to $\lambda$.

We may now state our main result in this section.

TheOREM 3.1. Let $T$ be a Markov operator on $L^{1}(X, \mathcal{B}, \lambda)$. Suppose its adjoint $U$ acting on $L^{\infty}(X, \mathcal{B}, \lambda)$ satisfies (iv). Assume moreover that the Markov operator $P: \mathcal{M}_{\text {fin }} \rightarrow \mathcal{M}_{\text {fin }}$ defined by (2.1) satisfies

$$
\left\|\left(P^{n} \mu\right)_{\mathrm{a}}\right\| \rightarrow 1 \quad \text { as } n \rightarrow \infty \text { for } \mu \in \mathcal{M}_{1} .
$$

Then $P$ is strongly asymptotically stable iff $T$ is asymptotically stable.

Proof. Let $\mu_{*} \in \mathcal{M}_{1}, P \mu_{*}=\mu_{*}$ and $\left\|P^{n} \mu-\mu_{*}\right\| \rightarrow 0$ for any $\mu \in \mathcal{M}_{1}$. We will show that $\left\{T^{n}\right\}$ is asymptotically stable. First by (A)

$$
\mu_{* \mathrm{a}}(X)=\left(P^{n} \mu_{*}\right)_{\mathrm{a}}(X) \rightarrow \mu_{*}(X) \quad \text { as } n \rightarrow \infty .
$$

Thus $\mu_{* \mathrm{~s}}=0$. Let $d \mu_{*}=u_{*} d \lambda$. Therefore $T u_{*}=d\left(P \mu_{*}\right) / d \lambda=d \mu_{*} / d \lambda=$ $u_{*}$. Further, for $u \in D$ let $d \mu_{n}=T^{n} u d \lambda$. Thus $\left\|T^{n} u-u_{*}\right\|_{1}=\left\|\mu_{n}-\mu_{*}\right\| \rightarrow 0$.

Conversely, assume that $\left\{T^{n}\right\}$ is asymptotically stable. Then there is $u_{*} \in D$ such that

$$
\left\|T^{n} u-u_{*}\right\|_{1} \rightarrow 0 \quad \text { for any } u \in D .
$$

Put $d \mu_{*}=u_{*} d \lambda$. Consider the sequence $\left\{P^{n} \mu\right\}$ with an arbitrary $\mu \in \mathcal{M}_{1}$. Choose $\varepsilon \geq 0$. According to (A) there exists an integer $k$ such that

$$
\left(P^{k} \mu\right)_{\mathrm{a}}(X)=\mu_{k \mathrm{a}}(X) \geq 1-\varepsilon .
$$

Define $\Theta=\mu_{k \mathrm{a}}(X)$. Since $\mu_{k}=\mu_{k \mathrm{a}}+\mu_{k \mathrm{~s}}$ we have

$$
\mu_{n+k}-\mu_{*}=P^{n} \mu_{k}-\mu_{*}=P^{n} \mu_{k \mathrm{a}}-\Theta \mu_{*}+P^{n} \mu_{k \mathrm{~s}}-(1-\Theta) \mu_{*}
$$

and so

$$
\left\|\mu_{n+k}-\mu_{*}\right\| \leq \Theta\left\|P^{n}\left(\Theta^{-1} \mu_{k \mathrm{a}}\right)-\mu_{*}\right\|+\left\|P^{n} \mu_{k \mathrm{~s}}\right\|+(1-\Theta)\left\|\mu_{*}\right\| .
$$

The last two terms are easy to evaluate. Namely,

$$
\begin{gathered}
\left\|P^{n} \mu_{k_{\mathrm{s}}}\right\|=P^{n} \mu_{k \mathrm{~s}}(X)=\mu_{k \mathrm{~s}}(X)=1-\Theta \leq \varepsilon, \\
(1-\Theta)\left\|\mu_{*}\right\|=(1-\Theta) \mu_{*}(X)=1-\Theta \leq \varepsilon .
\end{gathered}
$$

The measure $\Theta^{-1} \mu_{k \mathrm{a}}$ is absolutely continuous and normalized. Denote its density by $f_{\mathrm{a}}$. Evidently $P^{n}\left(\Theta^{-1} \mu_{k \mathrm{a}}\right)$ has density $T^{n} f_{\mathrm{a}}$ and by (3.3)

$$
\left\|P^{n}\left(\Theta^{-1} \mu_{k \mathrm{a}}\right)-\mu_{*}\right\|=\left\|T^{n} f_{\mathrm{a}}-u_{*}\right\|_{1} \rightarrow 0 .
$$

Combining this with (3.4)-(3.6) yields $\lim \left\|\mu_{n+k}-\mu_{*}\right\|=0$. 
4. The transport equation. Let $X=[0, \infty)^{n}$ with the Lebesgue measure $m$. We consider the integro-differential equation

$$
\frac{\partial u(t, x)}{\partial t}+\sum_{i=1}^{n} \frac{\partial}{\partial x_{i}}\left(F_{i}(x) u(t, x)\right)+u(t, x)=\int_{X} k(x, y) u(t, y) d y,
$$

$t \geq 0, x \in X$, with the boundary conditions

$$
\begin{gathered}
u\left(t, x_{1}, \ldots, x_{i-1}, 0, x_{i+1}, \ldots, x_{n}\right)=0, \quad i=1, \ldots, n, t \geq 0, \\
u(0, x)=f(x), \quad x \in X .
\end{gathered}
$$

We assume that $F_{i}$ has continuous derivatives $\partial F_{i} / \partial x_{j}, i, j=1, \ldots, n$, and that the solution of the equation

$$
\dot{x}(t)=F(x(t))
$$

with initial condition $x(0)=x^{0}$ exists for all $t \in \mathbb{R}$ for every $x^{0} \in \mathbb{R}^{n}$. This guarantees that (4.3) defines a group of transformations by

$$
\Pi\left(t, x^{0}\right)=x(t)
$$

where $x(t)$ is the solution of (4.3) with $x(0)=x^{0}$. By the well-known theorem on the continuous dependence of solutions of differential equations on the initial conditions, $\Pi: \mathbb{R} \times \mathbb{R}^{n} \rightarrow \mathbb{R}^{n}$ is a dynamical system. The kernel $k(x, y)$ is measurable and stochastic, i.e.,

$$
\int_{X} k(x, y) d x=1, \quad k(x, y) \geq 0, \quad x, y \in X .
$$

5. A linear evolution equation. In order to rewrite (4.1) as an evolution equation in $L^{1}$ space we must first replace the operator

$$
A \varphi(x)=-\sum_{i=1}^{n} \frac{\partial}{\partial x_{i}}\left(F_{i}(x) \varphi(x)\right)
$$

by its closure $A$ in $L^{1}$. We define

$$
D_{A}=\left\{\vartheta \in L^{1}\left(\mathbb{R}^{n}\right): \vartheta \text { is continuously differentiable }\right\} .
$$

Further, set

$$
I \vartheta=\vartheta, \quad K \vartheta(x)=\int_{X} k(x, y) \vartheta(y) d y .
$$

It is well known (see pp. 185-186 in [LaMa]) that $A$ is the generator of a semigroup $\left\{T_{0}(t)\right\}$ such that $u(t)=T_{0}(t) \vartheta$ satisfies the differential equation

$$
\dot{u}(t)=A u(t) \quad \text { for } t \geq 0 \text { and } \vartheta \in D_{A} .
$$

This semigroup is given by

$$
T_{0}(t) \vartheta(x)=1_{X}[\Pi(-t, x)] f(\Pi(-t, x)) \cdot J(-t, x),
$$


$\vartheta \in L^{1}$, where $J(-t, x)$ is the determinant of the Jacobian matrix of the transformation $x \mapsto \Pi(-t, x)$ and $1_{X}$ is the characteristic function of $X$.

The semigroup $\left\{T_{0}(t)\right\}_{t \geq 0}$ is continuous in $L^{1}$. That is, for every $\vartheta \in$ $L^{1}(X)$ the function $t \mapsto T_{0}(t) \vartheta$ is continuous in $L^{1}$ norm (see Remark 7.6.2 in [LaMa], p. 187). Analogously, $A-I$ is the generator of the semigroup $e^{-t} T_{0}(t)$ and $u=e^{-t} T_{0}(t) \vartheta$ for $\vartheta \in D_{A}$ is the solution of

$$
\dot{u}(t)=(A-I) u(t) .
$$

Finally, $A-I+K$ is the generator for the semigroup $\left\{T^{t}\right\}_{t \geq 0}$ of linear operators on $L^{1}$ such that $u=T^{t} \vartheta$ satisfies

$$
\dot{u}(t)=(A-I+K) u(t) \quad \text { for } \vartheta \in D_{A} .
$$

From the Phillips perturbation theorem [DuSc], $T^{t}$ is given by

$$
\left(T^{t} \vartheta\right)(x)=u(t, x)=e^{-t} \sum_{n=0}^{\infty} T_{n}(t) \vartheta(x)
$$

where

$$
T_{n+1}(t) \vartheta(x)=\int_{0}^{t} T_{0}(t-s) \circ K \circ T_{n}(s) \vartheta(x) d s .
$$

Thus instead of studying the solutions of (4.1) we shall study the behavior of the semigroup $\left\{T^{t}\right\}_{t>0}$.

The function $u(t)=T^{t} \vartheta$ may be considered as a generalized solution of (4.1). In fact, if $\vartheta \in D_{A}$, then $u(t)$ is a strong solution of (5.4) and for $k$ sufficiently smooth the formula $u(t, x)=T^{t} \vartheta(x)$ gives a classical solution of (4.1).

6. The Foias type solution of the transport equation. Let us summarize the properties of $\left\{T^{t}\right\}$ given by (5.5) and (5.6) which we will need in the sequel.

(a) $T^{t} \vartheta \geq 0$ for $\vartheta \geq 0, \vartheta \in L^{1}, t \geq 0$.

(b) $\left\|T^{t} \vartheta\right\|_{1}=\|\vartheta\|_{1}$ for $\vartheta \geq 0, \vartheta \in L^{1}, t \geq 0$.

From (a) and (b) it is easy to derive that

$$
\left\|T^{t} \vartheta\right\|_{1} \leq\|\vartheta\|_{1} \quad \text { for } \vartheta \in L^{1}, t \geq 0 .
$$

(c) $T^{t+s}=T^{t} \circ T^{s}$ and $T^{0}=I$ for $t, s \geq 0$.

(d) For every $\vartheta \in L^{1}$ the function $t \mapsto T^{t} \vartheta$ is continuous in $L^{1}$ norm.

From conditions (a) and (b) we have $T^{t} D \subset D$, where $D$ is the set of densities. 
Proceeding analogously to Section 2 we may define the semigroup $\left\{U^{t}\right\}_{t \geq 0}, U^{t}: L^{\infty}(m) \rightarrow L^{\infty}(m)$, of the adjoints to $T^{t}$. Namely,

$$
\left\langle T^{t} u, f\right\rangle=\left\langle u, U^{t} f\right\rangle \quad \text { for } u \in L^{1}(m), f \in L^{\infty}(m) .
$$

Then each $U^{t}$ satisfies conditions (i)-(iii) of Section 1. By a straightforward calculation the semigroup $\left\{U^{t}\right\}_{t>0}$ may be written explicitly in the form

$$
U^{t} f(x)=e^{-t} \sum_{n=0}^{\infty} U_{n}(t) f(x), \quad f \in L^{\infty},
$$

where

$$
\begin{gathered}
U_{n+1}(t) f(x)=\int_{0}^{t} U_{n}(s) P U_{0}(t-s) f(x) d s, \\
U_{0}(t) f(x)=f(\Pi(t, x)), \\
P f(y)=\int_{X} k(x, y) f(x) d x, \quad y \in X .
\end{gathered}
$$

Now we make the following additional assumption:

$$
P C(X) \subset C(X) .
$$

Lemma 6.1. Under assumption (B), the semigroup $\left\{U^{t}\right\}_{t \geq 0}$ given by (6.2) has the following property:

$$
U^{t} C_{0}(X) \subset C(X), \quad t \geq 0 .
$$

Proof. First we show that if $f$ is bounded then so is $U^{t} f$. Indeed, if $|f| \leq M$ then $\left|U^{t} f\right| \leq\left|U^{t} M\right| \leq M\left|U^{t} 1\right|=M$.

For fixed $f \in C(X), U_{i}(t) f(x)$ is continuous in $(t, x)$, since $U_{i}$ is a composition of transformations each of which carries a continuous function to a continuous one and the parameter $t$ appears in this composition when we compose a continuous function with $\Pi(t, x)$. Note, finally, that the series in (6.2) is convergent in the supremum norm with respect to $x$, is uniformly convergent with respect to $t$ on bounded intervals and thus $U^{t} f(x)$ is a continuous function of $(t, x)$.

Now we are in a position to define the family of Foiaş operators corresponding to the transport equation.

The mapping $P^{t}: \mathcal{M}_{\text {fin }}(X) \rightarrow \mathcal{M}_{\text {fin }}(X)$ given by

$$
P^{t} \mu(A)=\int_{X} U^{t} 1_{A} d \mu
$$

is called the Foiaş operator corresponding to $U^{t}$. 
We know that for every fixed $t \geq 0, P^{t}$ satisfies (I), (II) of Section 2 and moreover the family $\left\{P^{t}\right\}_{t \geq 0}$ forms a semigroup of Markov operators on $\mathcal{M}_{\text {fin }}$. That is,

(III) $P^{s+t}=P^{s} \circ P^{t}$ and $P^{0}=I$.

To prove this take $h \in C(X), h \geq 0$. There exists a sequence $\left\{h_{n}\right\}$, $h_{n} \in C_{0}(X)$, such that $h_{n} \uparrow h$. Then we have

$$
\left\langle h_{n}, P^{t} \mu\right\rangle=\left\langle U^{t} h_{n}, \mu\right\rangle, \quad \mu \in \mathcal{M}_{\text {fin }} .
$$

By (iii) for $U^{t}$ and the Lebesgue monotone convergence theorem

$$
\left\langle h, P^{t} \mu\right\rangle=\left\langle U^{t} h, \mu\right\rangle, \quad \mu \in \mathcal{M}_{\text {fin }} .
$$

To obtain (6.8) for every $h \in C(X)$, we put $h=h^{+}-h^{-}$, where $h^{+}(x)=$ $\max \{h(x), 0\}, h^{-}(x)=\max \{-h(x), 0\}$, and repeat the above argument for $h^{+}$and $h^{-}$.

Now, for $h \in C_{0}(X)$ and $\mu \in \mathcal{M}_{\text {fin }}$, from (6.8) we have

$$
\begin{aligned}
\left\langle h, P^{t+s} \mu\right\rangle & =\int U^{t+s} h d \mu=\int U^{t}\left(U^{s} h\right) d \mu=\left\langle U^{s} h, P^{t} \mu\right\rangle \\
& =\int U^{s} h d\left(P^{t} \mu\right)=\left\langle h, P^{s}\left(P^{t} \mu\right)\right\rangle .
\end{aligned}
$$

This proves (III).

Suppose that the initial value $u(0, x)$ in (4.2) is a measure $\mu_{0} \in \mathcal{M}_{\text {fin }}$. Then the semigroup $P^{t} \mu_{0}$ describes the evolution of the distribution $\mu_{0}$ with respect to $t$.

The semigroup $\left\{P^{t} \mu_{0}\right\}$ will be called the Foiaş solution of the transport equation (4.1) with initial condition $\mu_{0}$. Further, according to Section 2 observe that if a measure $\mu_{0}$ is absolutely continuous with respect to the Lebesgue measure $m$, that is, $d \mu_{0}=f_{0} d m, f_{0} \in L^{1}(m)$, then $P^{t} \mu_{0}$ is also absolutely continuous with respect to $m$ and

$$
d\left(P^{t} \mu_{0}\right)=\left(T^{t} f_{0}\right) d m,
$$

where $\left\{T^{t}\right\}$ is given by (5.5).

Analogously to Section 3 we say that $\left\{P^{t}\right\}$ is strongly asymptotically stable if there is a unique measure $\mu_{*} \in \mathcal{M}_{1}$ such that $P^{t} \mu_{*}=\mu_{*}$ for $t \geq 0$ and $\lim _{t}\left\|P^{t} \mu-\mu_{*}\right\|=0$ for every $\mu \in \mathcal{M}_{1}$. A semigroup $\left\{T^{t}\right\}$ of Markov operators is asymptotically stable if there is a unique $f_{*} \in D$ such that $T^{t} f_{*}=f_{*}$ for $t \geq 0$ and $\lim _{t}\left\|T^{t} f-f_{*}\right\|_{1}=0$ for every $f \in D$.

Now we are ready to state our main result concerning the strong asymptotic stability of the Foiass solutions of the transport equation.

THEOREM 6.1. The family of Foias operators $\left\{P^{t}\right\}$ is strongly asymptotically stable iff $\left\{T^{t}\right\}$ is asymptotically stable. In other words, the Foiaş solutions of the transport equation (4.1) are strongly asymptotically stable iff the solutions corresponding to densities are stable. 
Proof. An inspection of the proof of Theorem 3.1 shows that we only needed the following four requirements:

(1) $P$ satisfies conditions (I) and (II).

(2) $\left\{P^{n}\right\}$ is a semigroup on $\mathcal{M}_{\text {fin }}$, that is, $P^{n+m}=P^{n} \circ P^{m}$ and $P^{0}=I$.

(3) If $\mu \in \mathcal{M}_{\text {fin }}$ and $\mu$ is absolutely continuous with respect to $\lambda$, then so is $P^{n} \mu, n \geq 1$.

(4) $\left\{P^{n}\right\}$ satisfies condition (A).

Since our semigroup $\left\{P^{t}\right\}$ of Markov operators on $\mathcal{M}_{\text {fin }}$ satisfies analogous requirements to (1), (2) and (3) for every $t \geq 0$ with respect to the Lebesgue measure $m$, in order to adapt the proof of Theorem 3.1 to $\left\{P^{t}\right\}$ we must only prove condition (A) for the semigroup $\left\{P^{t}\right\}$. Namely, we will show that $\left(P^{t} \mu\right)_{\mathrm{a}}(X) \rightarrow 1$ as $t \rightarrow \infty$ for every $\mu \in \mathcal{M}_{1}$.

In order to evaluate $\left(P^{t} \mu\right)_{\mathrm{a}}$ we will use an explicit formula for $U_{n}(t) f$. From the recurrence formula for $U_{n}(t) f$, by a quite easy calculation, we may rewrite $U_{n}(t) f$ in the form

$$
\begin{aligned}
U_{n}(t) f(x)= & \int_{X} d w_{1} \ldots \int_{X} d w_{n} \int_{0}^{t} d s_{n} \int_{0}^{s_{n}} d s_{n-1} \ldots \\
& \ldots \int_{0}^{s_{2}} d s_{1} \varphi\left(t, s_{1}, \ldots, s_{n}, x, w_{1}, \ldots, w_{n}\right)
\end{aligned}
$$

where

$$
\begin{aligned}
\varphi\left(t, s_{1}, \ldots,\right. & \left.s_{n}, x, w_{1}, \ldots, w_{n}\right) \\
= & k\left(w_{1}, \Pi\left(s_{1}, x\right)\right) k\left(w_{2}, \Pi\left(s_{2}-s_{1}, w_{1}\right)\right. \\
& \quad \ldots \cdot k\left(w_{n}, \Pi\left(s_{n}-s_{n-1}, w_{n-1}\right)\right) \cdot f\left(\Pi\left(t-s_{n}, w_{n}\right)\right)
\end{aligned}
$$

and $s_{i} \in \mathbb{R}, w_{i} \in X, i=1, \ldots, n, 0 \leq s_{i} \leq s_{i+1}$.

Let $\mu \in \mathcal{M}_{1}$. Using the Lebesgue decomposition and linearity of $P^{t}$ we have $P^{t} \mu=P^{t} \mu_{\mathrm{a}}+P^{t} \mu_{\mathrm{s}}$. Note that $P^{t} \mu_{\mathrm{a}}$ is also absolutely continuous. Then by (II) we have

$$
\left(P^{t} \mu\right)_{\mathrm{a}}(X)=P^{t} \mu_{\mathrm{a}}(X)+\left(P^{t} \mu_{\mathrm{s}}\right)_{\mathrm{a}}(X)=\mu_{\mathrm{a}}(X)+\left(P^{t} \mu_{\mathrm{s}}\right)_{\mathrm{a}}(X) .
$$

Our goal now is to evaluate $\left(P^{t} \mu_{\mathrm{s}}\right)_{\mathrm{a}}(X)$. Let $A \in \mathcal{B}(X)$. By $(6.7)$

$$
P^{t} \mu_{\mathrm{s}}(A)=\int_{X} U^{t} 1_{A} d \mu_{\mathrm{s}}=e^{-t} \sum_{n=0}^{\infty} \int_{X} U_{n}(t) 1_{A} d \mu_{\mathrm{s}}=e^{-t} \sum_{n=0}^{\infty} P_{n}^{t} \mu_{\mathrm{s}} .
$$

Here

$$
P_{n}^{t} \mu_{\mathrm{s}}(A)=\int_{X} U_{n}(t) 1_{A} d \mu_{\mathrm{s}}=\int_{X} \nu\left(w_{n}, t\right) d w_{n}, \quad n \geq 1,
$$


where

$$
\begin{aligned}
\nu\left(w_{n}, t\right)= & \int_{X} \mu_{\mathrm{s}}(d x) \int_{X} d w_{1} \ldots \int_{X} d w_{n-1} \int_{0}^{t} d s_{n} \int_{0}^{s_{n}} d s_{n-1} \ldots \\
& \ldots \int_{0}^{s_{2}} d s_{1} \varphi_{A}\left(t, s_{1}, \ldots, s_{n}, x, w_{1}, \ldots, w_{n}\right)
\end{aligned}
$$

and $\varphi_{A}$ is obtained by substituting $f=1_{A}$ into (6.11). Thus

$$
P_{n}^{t} \mu_{\mathrm{s}}(A)=\int_{A} \nu\left(w_{n}, t\right) d w_{n}+\int_{X \backslash A} \nu\left(w_{n}, t\right) d w_{n}=\sigma_{t, n}(A)+\varrho_{t, n}(A) .
$$

The measure $\sigma_{t, n}(A)$ is absolutely continuous with respect to the Lebesgue measure with density $\nu\left(w_{n}, t\right)$. Hence

$$
\left(P_{n}^{t} \mu_{\mathrm{s}}\right)_{\mathrm{a}} \geq \sigma_{t, n} .
$$

Noting that $P_{n}^{t} \mu_{\mathrm{s}}(X)=\sigma_{t, n}(X)$ and using (6.13) we obtain

$$
\left(P_{n}^{t} \mu_{\mathrm{s}}\right)_{\mathrm{a}}(X)=P_{n}^{t} \mu_{\mathrm{s}}(X), \quad n \geq 0 .
$$

From this it follows that

$$
\begin{aligned}
\left(P^{t} \mu_{\mathrm{s}}\right)_{\mathrm{a}}(X) & \geq e^{-t} \sum_{n=1}^{\infty}\left(P_{n}^{t} \mu_{\mathrm{s}}\right)_{\mathrm{a}}(X)=e^{-t} \sum_{n=1}^{\infty} P_{n}^{t} \mu_{\mathrm{s}}(X) \\
& =P^{t} \mu_{\mathrm{s}}(X)-e^{-t} \mu_{\mathrm{s}}(X)=\mu_{\mathrm{s}}(X)-e^{-t} \mu_{\mathrm{s}}(X) .
\end{aligned}
$$

Thus $\left(P^{t} \mu_{\mathrm{s}}\right)_{\mathrm{a}}(X) \rightarrow \mu_{\mathrm{s}}(X)$ as $t \rightarrow \infty$. Finally, from (6.12) we have $\left(P^{t} \mu\right)_{\mathrm{a}}(X) \rightarrow 1$ as $t \rightarrow \infty$.

\section{References}

[DuSc] N. Dunford and J. T. Schwartz, Linear Operators, Part I, Interscience Publ., New York 1968.

[DłLa] T. Dłotko and A. Lasota, Statistical stability and the lower bound function technique, in: Semigroups, Theory and Applications, Vol. I, H. Brezis, M. Crandall and F. Kappel (eds.), Longman Scientific and Technical, 1987, 75-95.

[Klac] J. Klaczak, Stability of a transport equation, Ann. Polon. Math. 49 (1988), 69-80.

[LaMa] A. Lasota and M. C. Mackey, Probabilistic Properties of Deterministic Systems, Cambridge Univ. Press, 1985.

INSTITUTE OF INFORMATICS

JAGIELLONIAN UNIVERSITY

NAWOJKI 11

30-072 KRAKÓW, POLAND

Reçu par la Rédaction le 13.1.1992

Révisé le 18.2.1992 